\title{
Corrigendum: Mitotic internalization of planar cell polarity proteins preserves tissue polarity
}

\author{
Danelle Devenport, Daniel Oristian, Evan Heller and Elaine Fuchs
}

Nature Cell Biology 13, 893-902 (2011); published online 10 July 2011; corrected after print 12 January 2017

In the version of this Article originally published, Supplementary Figure 4c portrayed normal mitotic internalization of a Celsr1 $\Delta \mathrm{N}^{\text {STTTTS}}$ GFP mutant plasmid. Resequencing revealed an error in the sequence of this construct. Shown here are representative images of keratinocytes expressing a construct harbouring the correct sequence of this mutant (A). The bona fide mutant shows reduced mitotic internalization compared with the wild-type control (B). This error does not affect the data presented in the main paper, nor does it change the major conclusions drawn. Additionally, in light of this error, all of the other sequences of constructs used in this study were verified.

Supplementary Figure 4 has been corrected in the online versions of the Article and the text describing these data on page 897 has been changed accordingly.

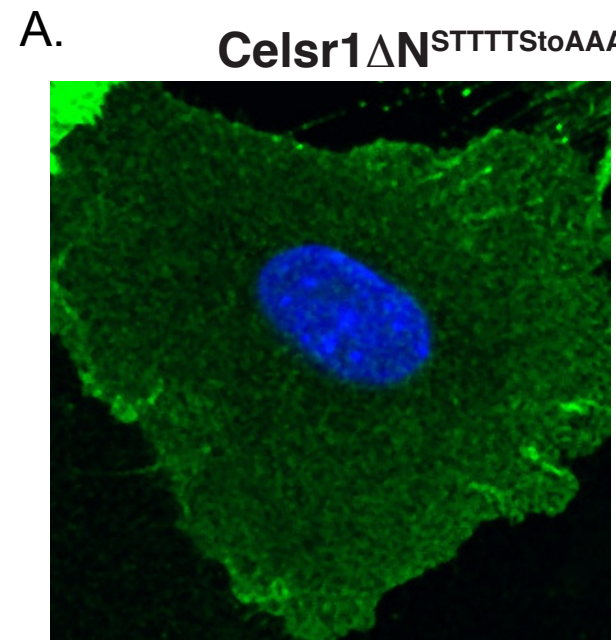

interphase

\section{AAA_GFP}

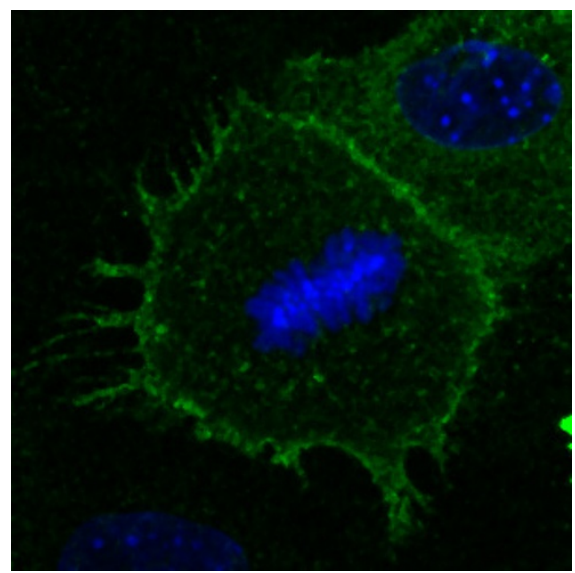

mitosis

\section{B. Celsr1 $\Delta \mathrm{N}^{\mathrm{WT}}$-GFP}

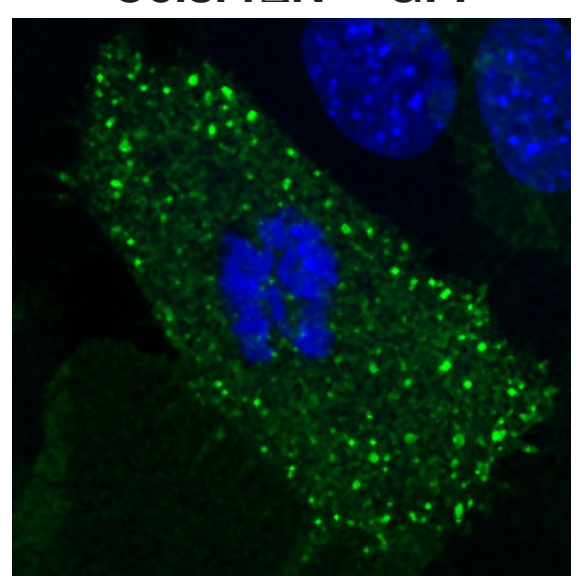

mitosis 